\title{
First Application of CVVHDF, Plasmapheresis and "Cytosorb Absorber" to Solve a Pediatric Haemophagocitic Histyocitosis Case
}

\author{
Milella $L^{*}$ and Ficarella MT \\ General Neonatal and Pediatric Anesthesia and Intensive Care Unit, Pediatric Hospital, Italy
}

*Corresponding author: Dr. L Milella, Director and Head, General Neonatal and Pediatric Anesthesia and Intensive Care Unit--Neonatal and Pediatric Cardiac Anesthesia and Intensive Care Unit, Pediatric Hospital “Giovanni XXIII”-Viale Amendola 207, 70121-Bari, Italy

Submission: 㭰 October 21, 2017; Published: 眥 December 08, 2017

\begin{abstract}
Hematologic filtration techniques increased very quickly in interest and application as adiuvant treatment for systemic Hyper inflammatory conditions. "High Level" mortality conditions as Septic Shock and Sepsis are still highly present if we consider the continuous therapeutical progress.

This is the clinical-therapeutic report of a female patient observed in September 2016, aged 4 , affected by HLH secondary to bacterial infection ; the patient also developed a secondary SDR with abnormal us macrophagic activation during septic shock and sepsis with MOF evidence status. The patient received mechanical controlled ventilation, strong cardiovascular drugs support, increase of volemia , CRRT, CVVHDF, intermittent plasmapheresis; a "Cytosorb Absorber" was added to the plasmapheresis circuit.

There was no Ethical Committee consent and the use of "Cytosorb", not approved for pediatrics weighting less than 40kgs, has been done in "Emminentia Mortis". We observed, after the first 12 hours of Conventional Hemodiafiltration plus Cytosorb application, a very rapid improvement of cardiovascular and respiratory patterns, a rapid decrease of hyperammonemia, improvement of renal and hepatic functionality, a quick decrease of inflammatory markers. The hemodiafiltration was continued, Cytosorb was added with a 24 hrs interval and Plasmapheresis treatment was continued. In a few days there was a normalization of clinical condition with resolution of sepsis and MOF.

Conclusion: There is a very little experience in the use of pediatric purification techniques especially in the use of Cytosorb. This is the first pediatric treatment with Hemodiafiltration, Plasmapheresis and Cytosorb in a case of a confirmed Hamofagocytic Histiocytosis disease. Further studies and informations are required.
\end{abstract}

Keywords: Hemofiltration techniques; Haemophagocityc Histyocitosis; Sepsis; Septic Shock; Cytosorb Absorber

\section{Introduction}

There is a huge interest in the use of Hematological Extracorporeal Purification The cliniques for unconventional treatment of the abnormal inflammatory response in case of Systemic Inflammatory Response Syndrom (SIRS), Sepsis, Septic Shock, MOF, Cardiac Acute failure; as we call it damages by "Cytokine Storm" [1].

Many clinical disorders can affect in the inflammatory response and conduct the patient to sepsis followed by MOF.

Acute and Chronic Cardiac Failure, decrease of $\mathrm{O}_{2}$ delivery and increase of $\mathrm{O}_{2}$ consumption, metabolic deficits, ARDS, Myocarditis, Pancreatitis, Acute Liver Failure, Hematologic Disease (leukemia, hemophagocytosis) and extracorporeal supportas cardiopulmonary By-pass in cardiac surgery, ECMO, VAD), especially in neonates and toddlers.
Sepsis is defined as an organ disfunction as consequence of a leading life infection; septic shock is a very dangerous aspect of sepsis; it is defined as an acute clinical condition in presence of high cardiovascular and metabolic failure; septic shock still presents a very high mortality, from $30 \%$ to $50 \%$ due to MOF. Septic shock is a very danger and precocious event wherever low peripheral perfusion condition is associated to high level of circulating cytokines [2].

The clinical triad Cardiac Failure, Vasoplegia, Organ Hypoperfusion defines a very dangerous situation where Metabolic Acidosis and Lactacidemia increase very fast. The use of catecholamines to support cardiac failure and vasoplegia can have a great influence in terms of morbidity as it may stabilize a self supporting dangerous clinical pathway related to an increase of peripheral low perfusion, metabolic acidosis and renal acute insufficiency. 
The first therapeutic target should be to reduce and trying to stop, in a very short time, the inflammatory cascade, to remouve the circulating cytokines, have a better control of the heart failure and consequent MOF, avoid the acute renal failure [3]. In the last decade many techniques have been developed and applied even to pediatrics and neonatal patients with the aim to achieve this result.

The High Volume Hemofiltration and Hemodiafiltration (HFVFHFVFD) was indicated as a good treatment for the removal from blood of cytochines and the reduction of inflammatory response but it was very difficult to apply this treatment to neonate and pediatric patients because of the high liquid volume of priming and flow rate. The absorber technique is less volume dependent and permits to reinfuse to the patient his own purified plasma volume. It also decreases the incidence of infection due to heterologous fluids, plasma and blood red cell replacement.

Cytosorbe absorber (Cytosorbents Europe GmbH, Berlin, Germany) is a new medical device for cytokines hematic absorbment approved in Europe for the use in patients weighting not less than $40 \mathrm{kgs}$. It consists of a highly porous polymer with a biocompatible surface conglobate in a filter for extracorporeal circulation. It has the capability to chain and remove a very high amount of hydrofobic components from blood having a molecular weight from 10 to $50 \mathrm{kDa}$; this range is comprehensive of the most part of cytokines.

Cytosorb doesn't remove all the plasmatic proteins but relates its action to molecular caliber and concentration. This can be consider a new deal in cytokines removal techniques because it is a very targeted treatment and seems to have a very fast action and effect $(<12$ hours you can observe a consistent decrease of cytokines and myoglobin levels)

Cytosorb use in neonatal and pediatric patients is not already permitted, there is no dedicate device and there are very few cases reported with the use of adult device. This is the first report of the use of a "Cytosorb Absorber" adopted in "Imminentia Mortis" during a non conventional therapeutic setting in a pediatric patient with a very rare and deadly disease $[4,5]$.

Hemophagocytic Histiocytosis are not malignant proliferative diseases, but they are characterized by an enormous increase of macrophagic cells during inflammatory response to infection. They have the tusk to process antigens. This is an aspect related to cytokines release de regulation as we can see in every macrophage activation syndrome [6].

The lymphocytic number increase is mainly due to TCD8 tissue in filtration, huge macrophagic increase and non controllable hypercytokinemia.

There are also present epatosplenomegalia, lymphadenopathy and Central Neurologic system compromission for the presence of activate macrophagic components in liquor and bone marrow and consequent coma and respiratory insufficiency.

Many times the diagnosis is a consequence of death and autoptic results. The disease is very difficult to have a rapid diagnosis because of the multiple and autoptic specific clinical presentations (neurological, respiratory, abdominal occlusion, renal impairement, SIRS and Shock, conclamate Sepsis non infection related)

Two different form of disease are present: Familial Hemophagocitic Lymphohistiocytosis (FHLH), and Hemophagocitic Histyocitosys (HLH). FHLH is an autosomic recessive disease having incidence of 1:50.000 birth with specific genomic alterations like the Porfirine, Munc-13 and Sintaxin mutation; the HLH is mostly secondary to infections, neoplasias and immunosuppressive therapy $[7,8]$.

The gender incidence is 1:1 between male and female. The two form are very difficult to recognize in advance, and surely the mechanism of action is reconductable, in the end, to an immunitary immunodeficiency that self sustains the dangerous clinical conditions and can drive very fast to death.

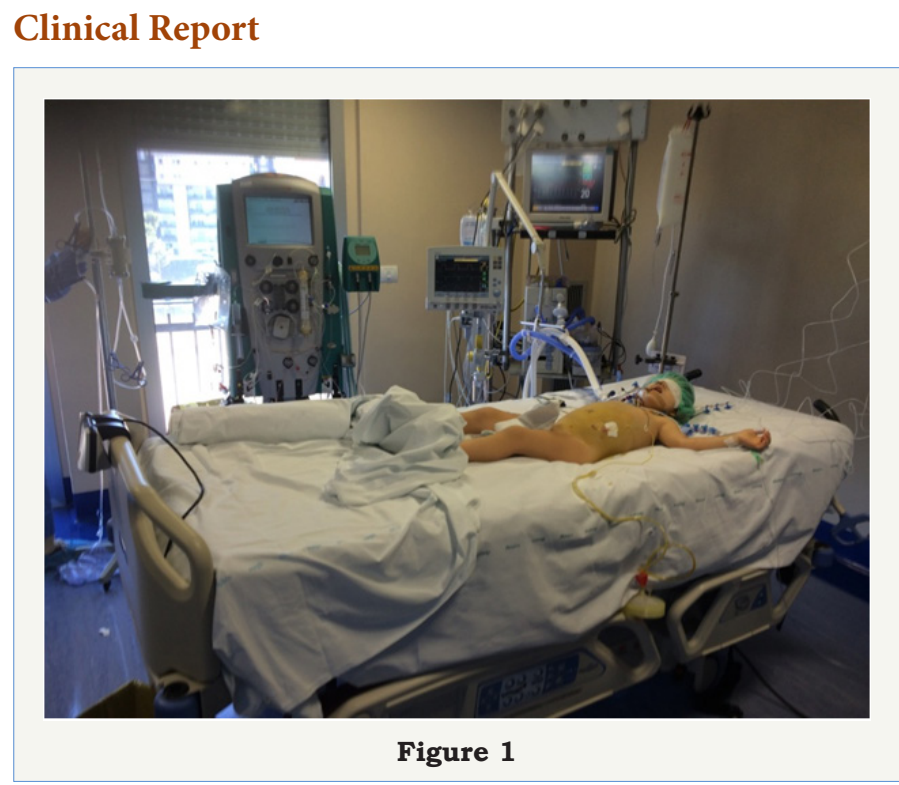

A little girl aged 4 with a weight of $19 \mathrm{kgs}$ came to our Pediatric ICU on 2016, July $31^{\text {st }}$ (Figure 1). She presented very critical clinical conditions. She was polyphonic, non responsive to stimulus, presented drawsiness, no finalistic responsiveness to pain stimulus; She was tachycardic (FC > 150bpm), presented hypotension (BP < $60 \mathrm{mHg}$ ) and cardiovascular instability; the abdomen was globosus and distended with clinical evidence of as cites, Icterus was present and oliguria was also present. Fever 38.4 Celsius Degrees. Sv02 value was $<50 \%$.mH

The laboratory tests defined leokucytosis (WBC 34.000; 86\%neutrophil), thrombocytosis (PLT 542.000) Anemia (Hb $8.5 \mathrm{gr} / \mathrm{dl}) \quad$ Hyperbilirubinemia $\quad(4,76 \mathrm{mg} / \mathrm{dl}$-mixed $50 \%) \mathrm{PCR}$ increase (12.8ng/l) PCT $(1 \mathrm{ng} / \mathrm{ml})$ hypoalbuminemia $(23,2 \mathrm{gr} / \mathrm{L}$ ) ALT $134 \mathrm{U} / \mathrm{L}-\mathrm{AST} 53 \mathrm{U} / \mathrm{L}, \mathrm{GGT} 253 \mathrm{U} / \mathrm{L}$, Lipase increase (1102 $\mathrm{U} / \mathrm{L}$ ), Amylase increase (257 U/L), Hyperammoniemia (74ug/ dl Hyper ferritinemia (538,70ng/l) normal PT-PTT INR but Hyperfibrinogenemia (364ng/L) and elevated D-Dimer value. Creatinine was normal.

Two days before the patient had received a video laparoscopic 
procedure because of abdominal pain, vomit and leukocytosis with fever; the procedure was negative for appendicitis and there was no evidence of surgical abdominal pathology. An important presence of as cites was noted but no obstruction in the portal district was defined.

An antibiotic therapy with cephalosporins and metronidazole was started. At the arrival of the patient in ICU oro-tracheal intubation and mechanical ventilation in $\mathrm{CPAP}+\mathrm{Ps}$ was started. Inotropic support with dobutamine was also started.

A complete viral and bacterial panel was required (PCR Tecnique) and performed, and parasitosys research was done. But there was no positive result except for the presence of Clostridium difficilis in feces at low title. There was a great positivity for presence of occult blood in feces [9-11].

The day after was noted an increase in as cites, hepato-pancreatic failure, increase ovmixed hyperbilirubinemia value and $39^{\circ} \mathrm{C}$ fever. It was decided to do a total body CT scan that showed a dilatation of intra hepatobiliar ways without alteration of hepatic structure, a very important increase of the as cites amount with retroperitoneal space diffusion, an augmented pancreatic dimension.

The lung CT scan showed different and diffuses bilateral atelectatic zones sited especially in posterobasal segments, presence of moderate pericardial effusion and a bilateral pleuric effusion. A diagnosis of initial pancreatitis was done by surgeon.

Albumine infusion and frusemide infusion were added to therapy and inothropic support with dobutamine was increased up to of $10 \mathrm{mcg} / \mathrm{kg} / \mathrm{min}$. A positivity for Streptococcus pneumonie in swabs was also found the same day.

A therapy with penicillin,teicoplanin and netilmicin was started but in a few hours the clinical conditions of the patient worsened and because of coma, respiratory insufficiency with hypoxia and hypercapnia, high fever more the $40{ }^{\circ} \mathrm{C}$ degrees, cardiovascular failure, oligoanuria persistent and finally anuria the patient had another ct scan showing a rapid worsening of lung parenchymal function, confirmed by laboratory data and there was the evidence of acclaimed MOF, with a sudden increase of the creatinine value to $3.7 \mathrm{mg} / \mathrm{dl}$. Ventilatory mechanical support was changed from assisted to controller in PCV, inotropic was changed from dobutamine to Noradrenaline and Adrenaline infusion.

\section{Materials and Methods}

A specific consent form for the use of Cytosorb has been signed from parents.

Before starting the hemofiltration procedure basal data were taken:

a. A viral and bacterial infection panel in blood, urine, groins and pharynx.

b. A peritoneal drainage and a culture of as CITIC liquid has been done; the drainage was left in situ in permanent draining.

c. A different antimicrobial therapy was started with
Cephalosporins $4^{\text {th }}$ generation, Metronidazole and Amikacine.

d. A dedicated 5,5F catheter - length $13 \mathrm{~cm}$, was positioned in right femoral vein percutaneously to start CRRT and CVVHDF.

\section{CVVHDF}

A CVVHDF continuous h 24was started using a Prismaflex Software Version 6.10 and a ST 60 Set CVVHDF Filter with priming of blood of $58 \mathrm{mls} /$ and a flow of $200 \mathrm{ml} / \mathrm{h}$. The dialysis liquid was Prismasol 2 with a QD from 1500 to $2000 \mathrm{ml} / \mathrm{h}$. Reinfusion at suspension of treatment was $600 \mathrm{ml}$. (es: filter clotting or filter changing).

Heparine infusion ranging from 10 to $30 \mathrm{IU} / \mathrm{kgh}$ was started and titrate with PTT-INR dosages and Thromboelastogram curve every eight hours. A negative pressure pump was setted to have a fluid rimotion in a range from 10 to $40 \mathrm{ml} / \mathrm{h}$.

Every six hour from 20 to $60 \mathrm{ml} / \mathrm{kg}$ of ultrafiltrate fluids was reinfused to the patient as specified from American College of Crirical Care (ACCC) using cristalloid fluids, fresh frozen plasma or PPS to asses the target of hematocrit value not more than $35 \%$ and a central venous pressure not less than $8 \mathrm{mmHg}$. This mode to control of infusion and removal of fluids set up and the use of inotropic drugs as Adrenaline and Noradrenaline titratre from 0,05 to $0,2 \mathrm{mcg} 7 \mathrm{~kg} / \mathrm{min}$, made the cardiovascular system more stable after a few hours.

\section{Plasmapheresis}

Plasmapheresis was performed every 24 hrs for 4 Hours, with suspension of CVVHDF and using the same Prismaflex software but a Prismaflex TPE 1000 Set with a blood priming of $67 \mathrm{ml}$ and exchange of 80-90 $\mathrm{ml}$ albumin/h (Figure 2).

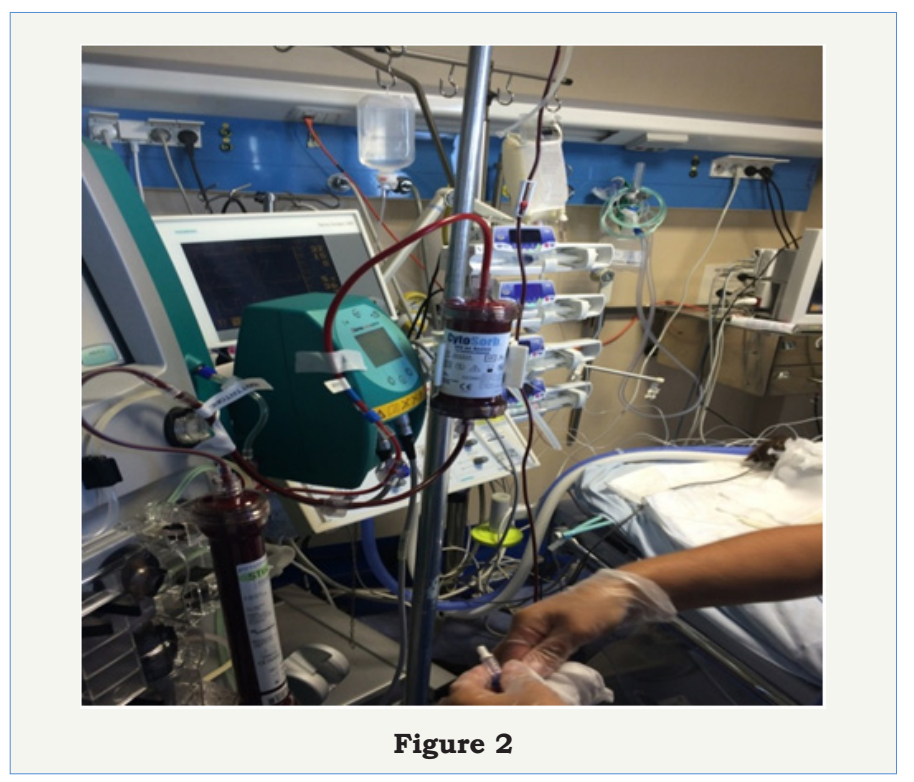

\section{Cytosorb application}

In the suspicious of a diagnosis of Hemophagocytic Histyocitosys Sindrome, CVVHDF was alternated with plasma pheresis with albumin as liquid exchanger. 
In consideration of the dramatic clinical condition of the patient was decided to add a" Cytosorb Absorbent" filter to the circuit in the aim to decrease the level of circulating cytockines in a faster way .

I. The Cytosorb filter was positioned on the CVVHDF standard circuit (Fresenius Medical Care, Bad Homburg, Germany) in a post dialysis filter site; the hematic blood priming of CVVHDF circuit was increased in volume because there was the necessity to rise up the original dialysis circuit flow of $200 \mathrm{ml} / \mathrm{min}$ with more $90 \mathrm{ml} / \mathrm{min}$.

II. The Cytosorb priming was $180 \mathrm{ml}$ and the flow was $90 \mathrm{ml} /$ $\min$

III. Cytosorb absorber adult device was added to the CVVHFD circuit and positioned immediately after the dialysis filter; a prime volume of $180 \mathrm{ml}$ was made with blood and a continuous flow of 90 $\mathrm{ml} / \mathrm{min}$ was maintained in addition to a median flow for CVVHFD of $200 \mathrm{ml} / \mathrm{min}$ (Figure 3).

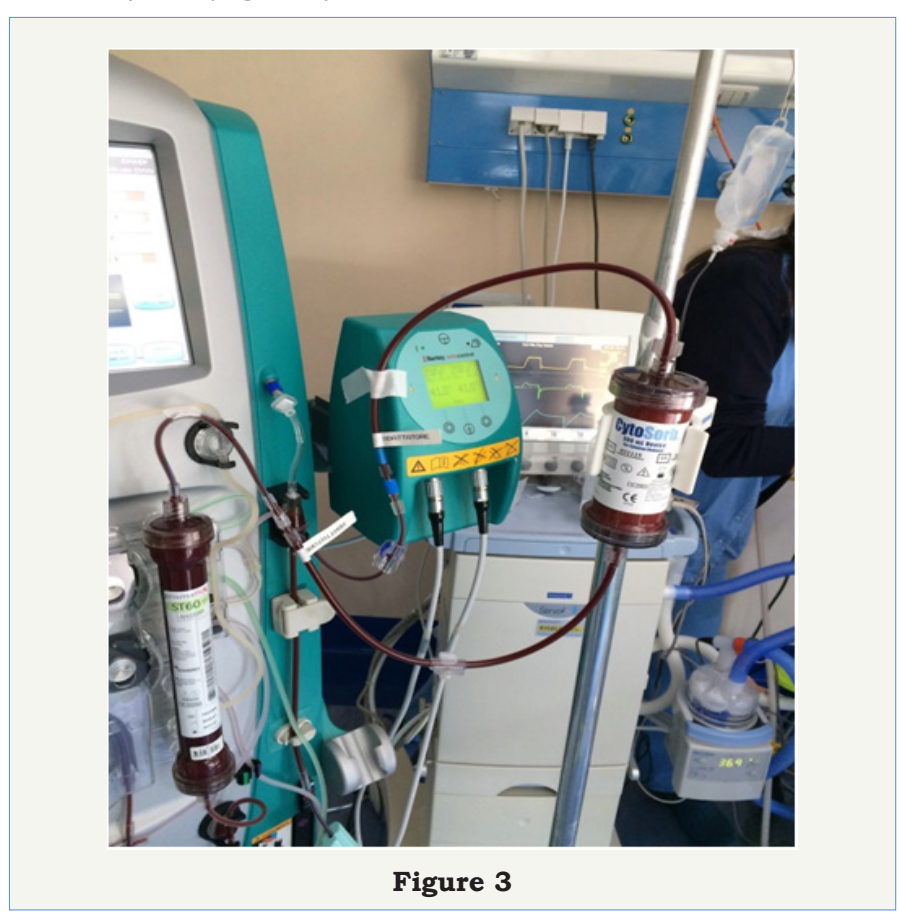

IV. The Cytosorb flow was maintained for 24 hours continuously.

No supplemental heparin was given (Table 1).

Table 1: Priming was done with blood.

\begin{tabular}{|c|c|c|c|}
\hline Tecniques & Primingmls & Flow mls/min & Duration-Days \\
\hline CVVHDF & 58 & $150-350$ & 5 \\
\hline Plasmapheresys & 67 & $1500-2000 \mathrm{mls} / \mathrm{h}$ & 5 \\
\hline Cytosorb & 180 & 90 & 3 \\
\hline
\end{tabular}

Before the Cytosorb was added to the circuit a serum blood sample was taken for basal Haematologic datas of Hemoglobin, Haematocrit, Free Haemoglobin, Myoglobin, Hemocrome Creatinine value, GOT and GPT and ammoniemia, Haemoagulative values.

Others two blood sample, one in sodium citrate and a second of serum, were taken a) These two samples were centrifugate and stored at less $80 \mathrm{C}$ degree for the dosage of citokines.

b) This scheme was maintained with samples taken every eight hours in the following $24 \mathrm{~h}$.

c) A final sample was taken soon after the suspension of the Cytosorb absorber use.

d) This Therapeutic scheme was continued for three times with a time interval of 24 hours. In between.

CVVHFD was continued for 5 days and a total of 5 plasmapheresis with albumin were done. The suspension of CVVHDF was related to a slowly but continuous reprise of a normal and efficient urinary output. We underline that during the extracorporeal treatment an infusion of frusemide $10 \mathrm{mg} / \mathrm{h}$ was never suspended.

After the first 24 hours of Cytosorb use there was the complete stabilization of cardiovascular status; an increase of the Svo 2 value that rised up from $59 \%$ to $79 \%$, a diminution of lactate production and of Bicarbonate deficit.

After three Cytosorb application the fluid replacement requirement diminished even during the plamapheresis time and CVVHDF, the PCR value fell down to $25 \mathrm{ng} / \mathrm{l}$ and the PCT was $0.2 \mathrm{ng} / \mathrm{ml}$. The dosage of vasoconstrictive drugs was reducted to half of initial dosage with a good mean arterial pressure and PCV pressure never less than 7-8 mmHg. 8 in fron of a total fluid intake of $45 / \mathrm{ml} / \mathrm{kg} x$ day. Ammoniemia became normal, WBC count was 17.000 , bilirubine value changed from 5.17 to $1.54 \mathrm{mg} / \mathrm{dl}$. Platelets count diminished from 400.000 to 45.000 and coagulative value as PTT-INR and PT-INR became normal with a still elevated value of D-Dimers but normalization of fibrinogen level. GOT and GPT returned normal with LDH total value at limits.

Diuresis returned normal (more than $3 \mathrm{ml} / \mathrm{kg} / \mathrm{h}$ ) and the creatinine value was at higher limit $1.2 \mathrm{ng} / \mathrm{l}$. The creatinine clearance still remained at 70\%VFR.

In fifth day CVVHDF and plasmapheresis were suspended and a repiratory weaning was started. Ascites was totally removed, there was no ecographic image of conclamate fluids in abdomen. The patient starting having no fever with an initial slope of temperature in third day. In this case, due to a deficit of specific tests, we didn't have any measure of circulating cytokines level before and after Cytosorb application.

We are overtaking this problem because all the samples, comprehensive of this case samples, are at the moment processed because we are driving a pilot study. In our opinion the clinical evidence and the specificity of the case seem to suggest that the use of Cytosorb was effective to solve a very complicated and preagonal situation at least in $72 \mathrm{hrs}$. The peritoneal drainage was removed in third day and enteral fasting started balanced with parenteral nutrition because of the reprise of peristaltis.

The ventilatory support was gently decreased and after the first 24 hours a of Cytosorb application a reduction of $\mathrm{FiO}_{2}$ from $80 \%$ to 
45\%; a riduction of PEEP from 7 to $4 \mathrm{mmHg}$ and of PIP from 18 to $15 \mathrm{mmHg}$ was possible. The $\mathrm{Po}_{2} / \mathrm{fiO}_{2}$ delta rised up from 150 to 350 $\mathrm{mmHg}$. The coma status was solved in third day with a reprise of a normal neurological status and a pediatric Glasgow score assessed from the initial value of 6 to 13 .

The respiratory weaning was started third day and according to a clear and not pathologic chest x-ray the patient was extubated in forth day; an assisted ventilation with Helmet Bubble CPAP with fiO2 $40 \%$ and PEEP of $5 \mathrm{~cm} /$ water was continued for two more days an than passed to High Flow CPAP ventilation in fourth day.

There was no positivity in the cultural samples and the suspect either of lympho myeloproliferative pathology or macrophagic activation syndrome raised up according with the presence of Hyperpyrexia, Hyperferritinemia, Hepatomegaly, Thrombocitopenia, Cholestasis, Neutrofilic Leucocicitosys.

A test was done finalized to a diagnosis of HLH or FHLH disease. A bone marrow needle aspiration was performed in forth day (negative). The as CITIC liquid culture was negative. The culture of cerebrospinal fluid was negative. In sixth day a diagnosis of HLH with a functional deficit of perforine, but without a gene mutagenesis, was done.

A cortisonic therapy with desametasone $20 \mathrm{mg} / \mathrm{m}^{2} /$ die was started and in tenth day the normalization of PCR $(4.5 \mathrm{ng} / \mathrm{l})$ PCT $(0,04 \mathrm{ng} / \mathrm{ml})$ WBC 15.000 , presepsine, hepatic and renal function, ammoniemia and no evidence of MOF was definitively demonstrated (Figure 4). In thirteen day the patient was dismissed from ICU to Oncoemathologic Paediatric Ward in very good and stable conditions (Figure 5) normally fasting and without any oxygen support (Figure 5).

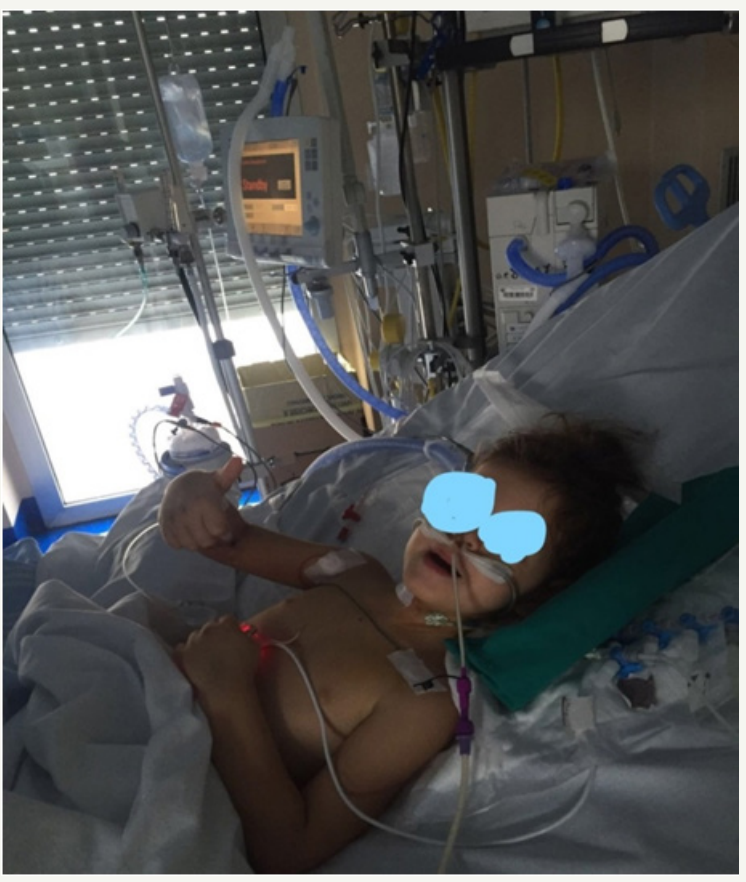

Figure 4

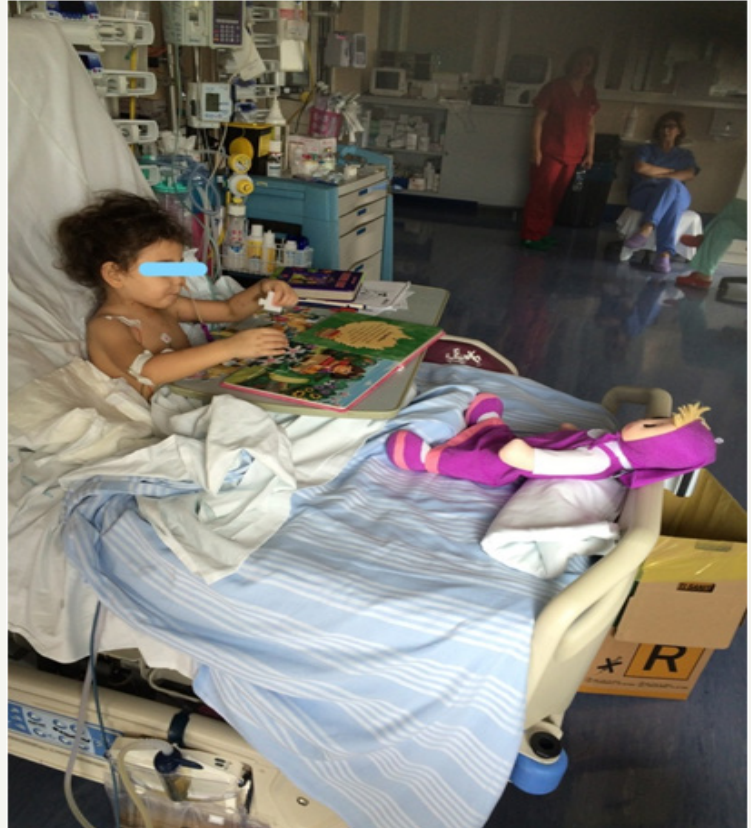

Figure 5

\section{Discussion}

This is the clinical-therapeutic report of a pediatric patient affected by "Secondary HLH" as consequence of infection with a transitory deficit of perforine that developed an acute sdr with secondary macrophage activation, septic shock, sepsis and MODS; she was treated with a combination a drugs and haemopurification techniques as CVVDHF, Plasmapheresis and the sperimental first time application of an adult absorber for rapidcitokines removal called Cytosorb.

There is a little letterature regarding the pediatric use of Cytosorb and there was non-related to the period whom we used it in "Imminentia Mortis".

There is an evidence of an increasing use of haemathologic purification with extracorporeal the coniques for the treatment of shock and septic shock but the main goal still remains the timing of application, the coagulation problems related to treatment and the impossibility to remove from blood in a very short time the cytokine and to stop in a very short time the so called "Cytikines Storm"

In neonatal and paediatrics this problem is strongly complicated from the difficulties to have dedicated matherials, filters and absorbers correlated to age, weight, organ maturation and volume of circulating fluids.

The absorber technique seems to be more reliable in the treatment of sepsis expecially when there is an increase of autoantibody factors, procoagulant factors, as ADAMATS-13, and cytokines causing an end Stage Liver Disease(ESLD).

Cytosorb was sperimentated and used to continue what has 
already been studied with The Prometheus System Device for extracorporeal support in liver transplant cases. It was selective for bilirubine removal.

In fact it worked as a Fractioned Plasma Separation and Adsorption.

Following the "Prometeus System" Cytosorb was prepared as an absorbption system able to remove susbstance with a molecular weight approximatively between 10 and $15 \mathrm{KDa}$.

This is because the major part of inflammatory response mediators, cytokines and interleukine, have this range of molecular weight. The perforine deficit is also associated to increase of interleukine 2, gamma interferon, alpha tumor necrosis factor.

The device also is able to remove myoglobin and bilirubine and bacterial derivate ditoxines as the staphilococcal endotoxin in TSST- 1 ; in the case we are presented there was no evidence of a bacterial or viral trigger infection and the clinical simpthoms were very inexpressive of a specific pathology, but, the laboratory data and the preagonal clinical situation required a very effective and rapid treatment in modulating the inflammatory response. There has been no good result from an conventional sepsis treatment and we decided, after the informed consent of parents, to add Cytosorb to CVVHDF and plasmapheresis in the aim to reduce the time of morbidity and action of inflammatory mediators trying to remove them in a massive amount in a shorter time.

It was a clinical decision confirmed by the evidence of a reduct perforine functional activity secondary to a pathogenic noxa and as we already said we confirm this data days after the critical moment. It was a life saving treatment in a case of very deadly pediatric disease, and we can assume in this specific case that Cytosorb use had a preciosus and effective role in the very rapid diminuition of cytokines and factors as IL10, IL6, TNF, complement active fractions as $\mathrm{C} 5 \mathrm{a}$, lactate level as it has already been demonstrated in report from adult use of Cytosorb.

Cytosorb seemed to be very helpful to solve the patient problem. If we consider the rapid progression in the respiratory recovery and extubation time and subsequent respiratory normal condition, the rapid recovery from coma, the very fast recovered cardiocircolatory stability, the resolution of as cites and the rapid (72hrs) improving of all the laboratory tests indicating the presence of a MOF, not least the rapid recovery of a spontaneous and adequate urinay production, we can rationally assume that the Cytosorb use was effective in treating the patient clinical problem in a very shortened time.
We are aware that a control of cytokines before and after every single treatment is compulsory and it is a part of the protocol we are at the moment applying for our pilot study (ETHICAL COMMITTEE 53891-2017) for the approval of the use of Cytosorb Absorber in neonatal and pediatric patients.

\section{Conclusion}

More study and the development of a dedicate neonatal and pediatric device are required to confirm that Cytosorb Tehecnology is "helpful" in removing the circulating inflammatory response mediators and that the system is able to do it in very rapid timing. Further studies are required.

\section{References}

1. Berkes A, Szikszay E, Kappelmayer J, Kerenyi A, Szabo T, et al. (2017) Use of Hemadsorption in a Case of Pediatric Toxic Shock Syndrome. Case Rep Crit Care 2017: 3818407.

2. FrieseckeS, Stecher SS, Gross S, FelixSB, Nierhaus A(2017) Extracorporeal Cytokine Elimination as Rescue Therapy in Refractory Septic Shock-a Prospective Single-Center Study. J Artif Organs 20(3):252-259.

3. Friesecke S, Trager K, Schitter GA, Molnar Z, Bach F, et al. (2017) International registry on the use of the CytoSorb ${ }^{\circledR}$ adsorber in ICU patients: Study protocol and preliminary results. Med Klin Intensivmed Notfmed.

4. Frimmer Marcel, Christine Nauk, Friedhelm Sayk, Sana Clinics Lubeck (2017) Use of CytoSorb in Streptococcus Pneumonia Sepsis. Cytosorb.

5. Fritsch Gerhard, Christian Schwarzwimmer (2017) Use of CytoSorb in Staph aureus Sepsis. Cytosorb.

6. Houschyar K, Pyles M, Rein S, Nietzschmann I, Duscher D, et al. (2017) Continuous Hemodiafiltration with a Cytokine-Adsorber During Sepsis a Review of the Literature. Int J Art Organs 40(5):205-211.

7. Klaus Kogelmann, Morten Scheller, DominikJarczak, Matthias Druner (2017) Successful treatment with CytoSorb in a case of septic shock, ARDS, multi organ failure and purpura fulminans due to acinetobacter baumannii pneumonia. 45(Suppl 1).

8. Klaus Kogelmann, Matthias Druner, Morten Scheller (2017) Use of CytoSorb in a septic shock patient with pneumonia, gangrenous cholecystitis and suspicion of HLH. Cytosorb.

9. Lennart Bartolitius, Holger Brammer, Peter Rosenthal, Andreas Schuchert (2017) CytoSorb in septic shock with multiple organ failure and ARDS.

10. Marek S, Gamper G, Reining G, Bergmann P, Mayr H, et al. (2017) ECMO and cytokine removal for bridging to surgery in a patient whit ischemic ventricular septal defect -a case report. Int J Artif Organs 40(9): 526529.

11. Rybalko AS, Pytal AV, Tepaev RF (2017) Use of CytoSorb in a pediatric patient with severe SIRS, ARDS and vasoplegia post-cardiac surgery. Cytosorb. 